\section{Efectos de un programa de entrenamiento de marcha sobre las capacidad aeróbica y la función cognitiva en mujeres diabéticas de edad avanzada}

\author{
EDGARDO MOLINA-SOTOMAYOR ${ }^{1}$, ROSSANA GÓMEZ-CAMPOS ${ }^{2}$ \\ EDUARDO ULLOA-TAPIA ${ }^{3}$, PATRICIO ARROYO-JOFRE ${ }^{4}$, \\ JOSÉ GONZÁLEZ-JURADO ${ }^{5}$, CARLOS CELIS-MORALES $^{6}$, \\ FERNANDO RODRÍGUEZ-RODRÍGUEZ7 ${ }^{7}$, MARCO COSSIO-BOLAÑOS²
}

\section{Effects of physical exercise on aerobic fitness and cognition in older women with type 2 diabetes mellitus}

Background: Among older people, physical exercise improves cognitive function, aerobic fitness, and thus functional independence. Aim: To determine the effects of a walking training program on aerobic fitness and cognitive function in older women with type 2 diabetes mellitus. Material and Methods: An experimental study was carried out in 76 women with type 2 diabetes mellitus aged between 64 and 78 years. Thirty-eight women in the exercise group (EG) participated in a controlled walking program 3 times a week for 48 sessions (60min /day) and 38 women in the control group (CG) were not trained. Weight, height, body mass index (BMI), estimated maximal oxygen uptake ( $\mathrm{VO}_{2}$ max), and cognitive function using the Minimental test were evaluated at baseline and the end of the intervention. Results: The Minimental test improved significantly in the exercise group and did not change in the control group. Estimated VOmax improved in women aged between 69 and 78 years. The distance walked in 6 minutes increased in all women of the experimental group. No changes in these parameters were observed in the control group. Conclusions: A structured walking program improved cognitive function, estimated aerobic capacity, and walking distance in these diabetic women.

(Rev Med Chile 2021; 149: 37-44)

Key words: Cognition; Diabetes Mellitus; Exercise; Frail Elderly.

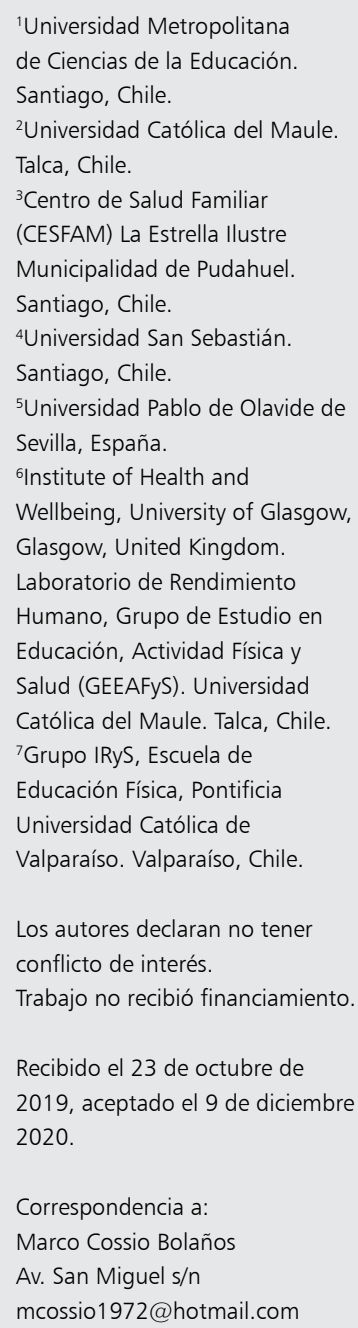

L a disminución de la función cardiorrespiratoria y el rendimiento muscular asociado con la edad avanzada y/o la inactividad pueden disminuir significativamente la capacidad funcional de un individuo de edad avanzada ${ }^{1}$. Muchas de estas personas mayores tienen al menos una afección médica crónica, si no múltiple, como artritis, hipertensión o diabetes ${ }^{2}$.

Actualmente Chile es un País que se encuentra en un proceso de transformación demográfica y de modificación de estilo de vida, que ha conducido a un incremento en las tasas de prevalencia de diabetes mellitus y obesidad más altas del continente, representado $11 \%$ de la población adulta entre 20-79 años .

De hecho, el mantenimiento de las funciones cognitivas durante el envejecimiento es un requisito previo para la independencia funcional, calidad 
de vida, la salud en general ${ }^{4}$. También se ha demostrado que la diabetes tipo 2 está asociada con trastornos vasculares, inclusive se ha considerado como un factor de riesgo independiente sobre el deterioro cognitivo y la demencia senil ${ }^{5}$.

Algunos estudios destacan que la práctica regular del ejercicio físico puede impartir beneficios neurológicos y efectos protectores sobre la capacidad y desempeño cognitivo, previniendo el deterioro a lo largo del envejecimiento ${ }^{6,7}$. Además, es ampliamente conocido que el ejercicio físico aeróbico es esencial para contrarrestar el sobrepeso y obesidad, e incrementar la aptitud aeróbica en diversas poblaciones ${ }^{8,9}$.

En general, el ejercicio físico regular y el aumento de la aptitud aeróbica se asocian con una disminución en la mortalidad y morbilidad por todas las causas en los adultos de mediana y edad avanzada $^{10}$.

Entre los tipos de actividad física específica y de bajo coste que se suelen realizar como parte del ocio, se considera la caminata. Este tipo de actividad es cada vez más utilizado como un medio de promoción de la salud y de prevención de enfermedades en los adultos mayores ${ }^{11} \mathrm{y}$ juega un papel relevante en la participación de conductas de estilo de vida saludables (actividad física, dieta, estimulación intelectual, compromiso social), previniendo o retrasando el deterioro cognitivo relacionado con la edad y/o la aparición de demencia $^{7,12}$ e inclusive contribuye al mejoramiento en la independencia funcional, capacidad cardiovascular, riesgo de diabetes, y previene el riesgo de caídas ${ }^{13-15}$.

En consecuencia, basados en que la actividad física y específicamente donde la caminata presenta un valor potencial en las actividades cotidianas en los adultos mayores, y donde el entrenamiento prolongado a intensidades aeróbicas moderadas, da como resultado adaptaciones fisiológicas, que incluyen aumento del volumen sanguíneo, densidad capilar, tamaño y densidad mitocondrial, mejor movilización de la grasa y termorregulación, lo que en su conjunto representan una mejora de la condición cardiorrespiratoria ${ }^{16}$, este estudio hipotetiza que la práctica sistemática de la caminata de moderada a vigorosa intensidad podría producir cambios significativos sobre el estado cognitivo EC, e inclusive podría mejorar la aptitud aeróbica de mujeres de tercera edad con diabetes mellitus de tipo 2 .
El objetivo principal del estudio es determinar si la práctica cotidiana de la caminata controlada sistemáticamente influye positivamente sobre el EC y la aptitud aeróbica de mujeres adultas de la tercera edad con diabetes mellitus tipo 2 .

\section{Material y Métodos}

\section{Tipo de estudio y muestra}

Se efectuó un estudio experimental en 76 mujeres de tercera edad con diabetes mellitus tipo 2, con diagnóstico de deterioro cognitivo leve (DCL), con un puntaje total $\leq$ a 24 puntos, valorado por el Mini Mental State Examination $(\mathrm{MMSE})^{17}$. Las mujeres fueron reclutadas de un programa de actividad física de un Centro de Salud Familiar de la Región Metropolitana de Santiago (Chile). Una vez inscritas ( $\mathrm{n}=90$ mujeres) se aleatorizaron en dos grupos (grupo experimental GE $\mathrm{n}=45$, y grupo control GC $\mathrm{n}=45$ ) por medio de randomización, llegando a conformar dos grupos equivalentes.

Al final de las evaluaciones e intervención los grupos quedaron conformados por 38 mujeres GE y 38 mujeres GC. La Figura 1 muestra la organización del diseño experimental puro.

Se incluyeron en el estudio a todas las mujeres que presentaban diabetes diabetes mellitus tipo $2 \mathrm{y}$ las que demostraron tener un nivel de alfabetización que les permitía leer y escribir sin ayuda. Se excluyeron a las mujeres que no se encontraban en el rango de edad establecido y a las que no cumplieron con el $85 \%$ asistencia obligatoria al programa de intervención. El estudio contó con la aprobación del comité de ética de la Universidad Pablo de Olavide (España). Se efectuó respetando el acuerdo de Helsinki para seres humanos.

\section{Procedimientos}

Las evaluaciones del pre test y post test de ambos grupos se efectuaron en un laboratorio cerrado de la Universidad (UMCE, Chile). Se evaluó inicialmente la evaluación de la función cognitiva, seguido de la evaluación antropométrica y la aptitud aeróbica.

Los procedimientos de evaluación estuvieron a cargo de tres profesionales (autores del artículo), donde cada uno se hizo responsable de una variable. Las evaluaciones se efectuaron en el período de la mañana entre las 8:30 a 10:00 am durante una semana antes y después de la intervención. 




Figura 1. Organización del diseño experimental.
El estado cognitivo (EC) fue evaluado por medio del test propuesto por Folstein et $\mathrm{al}^{18}$ denominado Mini Mental State Examination (MMSE). La versión en español fue validada en Chile por Quiroga et $\mathrm{al}^{17}$. El test fue aplicado en forma individual en una sala del Centro de Salud Familiar. A cada paciente se le explicó y se hizo lectura de las instrucciones del test, registrando nombre edad y nivel de escolaridad, además se les indicó que no había límite de tiempo para completar el test.

Para la evaluación de la masa corporal $(\mathrm{kg})$ se utilizó una balanza electrónica (United Kingdom, Ltd) con una precisión de $100 \mathrm{~g}$. La estatura $(\mathrm{cm})$ se evaluó por medio de un estadiómetro (Seca Gmbh \& Co. KG, Hamburg, Germany) con una precisión de $1 \mathrm{~mm}$. El Índice de masa Corporal IMC fue calculado por la fórmula: [IMC = Masa Corporal $(\mathrm{kg}) /$ Estatura $\left.(\mathrm{m})^{2}\right]$.

La evaluación de la glucosa se efectuó siguiendo las recomendaciones de la Asociación Americana de la Diabetes ${ }^{19}$. Este procedimiento estuvo a cargo del personal sanitario del Centro de Salud. Una enfermera tomó la muestra de sangre venosa periférica en ayunas (8-10 h de ayuno). La punción se hizo en la vena mediana cubital del brazo derecho, extrayéndose $10 \mathrm{ml}$, que fueron vertidos en tubos de muestras al vacío, mezclando con gel inerte, para su conservación y posterior análisis.

La aptitud aeróbica se evaluó por medio de una prueba de campo indirecta descrita por Mossberg,
Fortini ${ }^{20}$, puesto que es una prueba que se utiliza habitualmente para evaluar a pacientes con enfermedades cardiopulmonares, insuficiencia cardíaca congestiva y diabetes ${ }^{21}$. Para calcular el $\mathrm{VO}_{2}$ se utilizó la fórmula del Colegio Americano de Medicina del Deporte ${ }^{22}: \mathrm{VO}_{2}(\mathrm{ml} / \mathrm{kg} / \mathrm{min})=(0,2 \times \mathrm{V})+3,5$. El tiempo se expresó en minutos y la distancia en metros. La evaluación de la distancia se efectuó en una pista de atletismo de $400 \mathrm{~m}$, donde el sujeto caminó 6minutos. Esta prueba está diseñada para caminatas a diferentes velocidades. La evaluación se distribuyó en grupo de 5 pacientes, cada sujeto se ubicó en un carril y las distancias de los $400 \mathrm{~m}$ fueron marcadas a cada $50 \mathrm{~m}$ para facilitar el registro de los metros recorridos.

\section{Programa experimental}

El entrenamiento de caminata, consistió en caminar progresivamente en plano horizontal a diferentes porcentajes del $\mathrm{VO}_{2 \max }$. Las intensidades del esfuerzo durante los mesociclos oscilaron desde 40 hasta $60 \%$. Las velocidades de las caminatas se efectuaron desde 52,2 metros/minuto hasta $102 \mathrm{~m} / \mathrm{min}$ (Figura 2). La frecuencia fue de 3 veces por semana, cada sesión fue de $60 \mathrm{~min} /$ día, con dos descansos de $5 \mathrm{~min}$, cada sesión. Antes de iniciar cada sesión de caminata se efectuó un calentamiento y ejercicios de flexibilidad que duraban entre 10-15 min. El programa tuvo 4 meso-ciclos (cada uno con 4 semanas) totalizando 48 sesiones. 


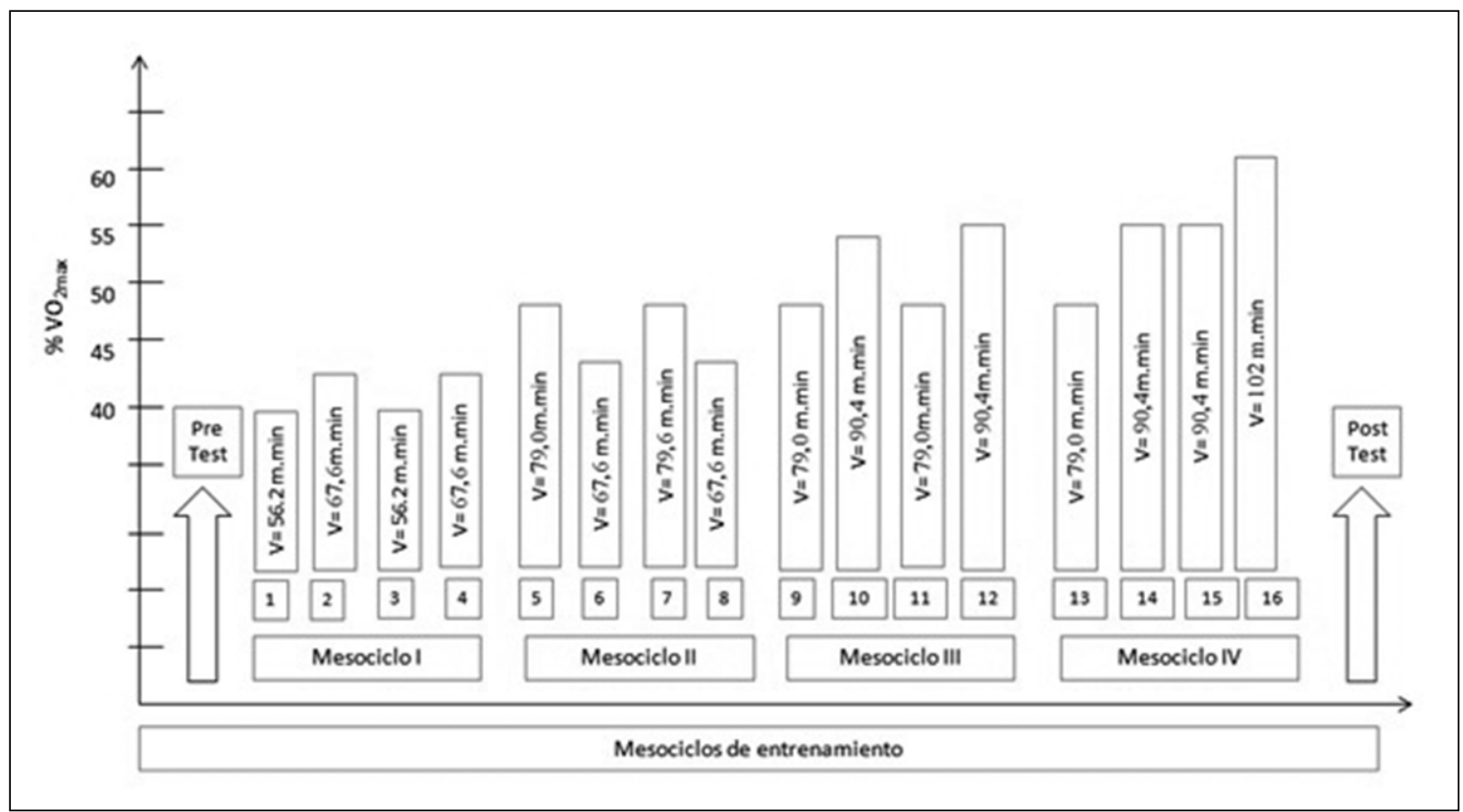

Figura 2. Protocolo utilizado para la organización de las caminatas, según $\%$ de $\mathrm{VO}_{2 \max }$ y velocidad de caminata.

La Figura 2, ilustra el protocolo del programa de intervención.

\section{Estadística}

La normalidad de los datos se verificó por medio de la prueba de Shapiro Wilk. Se utilizó la estadística descriptiva de media aritmética, desviación estándar, intervalo de confianza para caracterizar ambos grupos de estudio. Las diferencias entre el pre y post test se verificaron por medio de Anova de dos vías y la prueba de especificad de Tukey. Se determinó el tamaño del efecto (d) para los cambios entre los valores de pre test y post test tanto para la EC como para el $\mathrm{VO}_{2}$ max, de acuerdo con Cohen ${ }^{23}$. Todo el análisis estadístico se efectuó en SPSS 16,0 para Windows, adoptando un nivel de significación de $5 \%$.

\section{Resultados}

Las características de ambos grupos de estudio (GE y GC) se pueden observar en la Tabla 1. Nótese que entre ambos grupos no se observó diferencias significativas en la edad, variables antropométricas y los niveles de glucosa $(\mathrm{p}<0,05)$.

Las comparaciones del EC de ambos grupos de estudio se observan en la Tabla 2. Se ha determinado diferencias significativas entre el post test de ambos grupos $(\mathrm{p}<0,05)$ en los tres rangos de edad y en todo el grupo estudiado. Además, hubo diferencias significativas entre el pre y post test del GE $(p<0,05)$ y el tamaño del efecto ( $d$ de Cohen) fue moderado oscilando entre 0,31 a 0,49 . Por el contrario en el GC no hubo diferencias significativas $(p>0,05)$ entre el pre y post test, con un tamaño de efecto pequeño $(\mathrm{d}<0,20)$.

En relación a la aptitud aeróbica, como se muestra en la Tabla 3, no se observó diferencias significativas en el primer rango de edad (64-68 años) y en todas las edades entre los post test de ambos grupos e inclusive entre el pre y post test del GE ( $p>0,05)$, en todos los casos el tamaño de efecto fue pequeño $(d<0,20)$. Por el contrario, se observó diferencias significativas entre los post test de ambos grupos (GE y GC), tanto en el segundo rango de edad (69-73 años) con un tamaño de efecto moderado $(\mathrm{d}=0,515)$, como para el tercer rango de edad (74-78 años), con moderado tamaño de efecto $(\mathrm{d}=0,58)$, además en el GE hubo diferencias entre el pre y pos test de ambos rangos de edad $(\mathrm{p}<0,05)$ con un tamaño de efecto pequeño $(d<0,20)$. 
Ejercicio físico sobre aptitud aeróbica en mujeres mayores - E. Molina-Sotomayor et al

Tabla 1. Características antropométricas y de glucosas de ambos grupos de estudio $(n=76)$.

\begin{tabular}{|c|c|c|c|c|c|c|c|c|c|}
\hline \multirow[t]{2}{*}{ Variables } & \multicolumn{3}{|c|}{ GC $(n=38)$} & \multicolumn{3}{|c|}{ GE $(n=38)$} & \multicolumn{3}{|c|}{ Total $(n=76)$} \\
\hline & $\overline{\mathbf{x}}$ & DE & IC & $\overline{\mathbf{x}}$ & DE & IC & $\overline{\mathbf{x}}$ & DE & IC \\
\hline Edad (años) & 72,2 & 4,3 & $70,7-73,6$ & 72,6 & 3,9 & $71,4-73,8$ & 72,4 & 4,1 & $71,5-73,3$ \\
\hline Peso (kg) & 79,8 & 6,0 & $77,8-81,8$ & 80,1 & 7,3 & $77,8-82,3$ & 80,0 & 6,7 & $78,5-81,4$ \\
\hline Estatura $(\mathrm{cm})$ & 159,0 & 4,8 & $157,5-160,7$ & 160,0 & 5,1 & $158,4-161,6$ & 160,0 & 4,9 & $158,5-160,7$ \\
\hline IMC $\left(\mathrm{kg} / \mathrm{m}^{2}\right)$ & 31,1 & 2,2 & $30,4-31,9$ & 31,0 & 3,5 & $29,9-32,1$ & 31,1 & 3,0 & $30,4-31,7$ \\
\hline Glucosa (mg/dl) & 125,0 & 14,0 & $120,1-129,1$ & 125,0 & 19,0 & $119,0-131,0$ & 125 & 17,0 & $121,1-128,6$ \\
\hline
\end{tabular}

GC: Grupo control, GE: grupo experimental.

Tabla 2. Comparación de los valores medios y \pm DE de la EC del GC y GE durante el pre y post test

\begin{tabular}{|c|c|c|c|c|c|c|c|c|}
\hline \multirow{3}{*}{$\begin{array}{l}\text { Edades } \\
\text { (años) }\end{array}$} & \multicolumn{4}{|c|}{ GC } & \multicolumn{4}{|c|}{ GE } \\
\hline & \multicolumn{2}{|c|}{ Pre test } & \multicolumn{2}{|c|}{ Pos test } & \multicolumn{2}{|c|}{ Pre test } & \multicolumn{2}{|c|}{ Pos test } \\
\hline & $\overline{\mathbf{x}}$ & DE & $\overline{\mathbf{x}}$ & DE & $\overline{\mathbf{x}}$ & DE & $\overline{\mathbf{x}}$ & DE \\
\hline 64 a 68 & 22,8 & 0,7 & 22,3 & 0,9 & 23,2 & 0,4 & $24,20^{a^{*}}$ & 1,3 \\
\hline 69 a 73 & 23,3 & 0,6 & 22,9 & 1 & 23,1 & 0,8 & $24,10^{a^{*}}$ & 1,3 \\
\hline 74 a 78 & 23,2 & 0,8 & 23,1 & 1,3 & 23,1 & 0,8 & $24,20^{a^{*}}$ & 1,1 \\
\hline Todos & 23,1 & 0,7 & 22,9 & 1,2 & 23,1 & 0,7 & $24,10^{a^{*}}$ & 1,2 \\
\hline
\end{tabular}

${ }^{a}$ Diferencia significativa en relación al pos test del GC; *diferencia significativa en relación al pre test del GE.

Tabla 3. Comparaciones de los valores medios y $\pm \mathrm{DE}$ del $\mathrm{VO}_{2 \max }$ del pre test y post test de ambos grupos estudiados

\begin{tabular}{|c|c|c|c|c|c|c|c|c|c|c|}
\hline \multirow[t]{3}{*}{ Edades (años) } & \multicolumn{5}{|c|}{ GC } & \multicolumn{5}{|c|}{ GE } \\
\hline & \multirow[t]{2}{*}{$\mathbf{n}$} & \multicolumn{2}{|c|}{ Pre test } & \multicolumn{2}{|c|}{ Pos test } & \multirow[t]{2}{*}{$\mathbf{n}$} & \multicolumn{2}{|c|}{ Pre test } & \multicolumn{2}{|c|}{ Pos test } \\
\hline & & $\overline{\mathbf{x}}$ & DE & $\overline{\mathbf{x}}$ & DE & & $\overline{\mathbf{x}}$ & DE & $\overline{\mathbf{x}}$ & DE \\
\hline 64 a 68 & 8 & 19,1 & 1,5 & 18,6 & 1,1 & 6 & 19,8 & 2,1 & 21 & 2,1 \\
\hline 69 а 73 & 11 & 19,2 & 1,1 & 18,4 & 1,2 & 14 & 19,4 & 1,5 & $20,30^{\mathrm{a}^{*}}$ & 2 \\
\hline 74 a 78 & 19 & 19,3 & 1,8 & 18,6 & 1,5 & 22 & 19,2 & 1,5 & $20,40^{\mathrm{a}^{*}}$ & 1,6 \\
\hline Todos (ml/kg/min) & 38 & 19,2 & 1,5 & 18,6 & 1,3 & 42 & 19,4 & 1,6 & 20,5 & 1,8 \\
\hline Todos (metros) & 38 & 407 & 62 & 402 & 76 & 42 & 546,9 & 42 & $572,1^{\mathrm{a}^{*}}$ & 43 \\
\hline
\end{tabular}

aDiferencia significativa en relación al pos test del GC; * diferencia significativa en relación al pre test del GE.

\section{Discusión}

Los resultados del estudio han evidenciado que la caminata controlada iniciada desde el $40 \%$ hasta el $60 \%$ del $\mathrm{VO}_{2 \max }$ durante 48 sesiones de entrenamiento de una hora, fueron suficientes para mejorar significativamente el EC y la aptitud aeróbica de mujeres de la tercera edad con diabetes mellitus tipo 2.

Estos hallazgos son consistentes con algunos estudios que han utilizado la caminata como intervención para mejorar la cognición ${ }^{24,25} \mathrm{y}$ aptitud aerobica $^{26,27}$ de adultos mayores, independientemente del tipo de dolencia.

En consecuencia, al parecer el EC y la aptitud aeróbica mejoraron debido a los efectos positivos que produce el ejercicio (caminata) sobre el flujo sanguíneo cerebral $^{28}$, además, los mejores niveles de aptitud aeróbica pueden estar asociados con un aumento del volumen del hipocampo, lo que 
podría conducir a un mejor rendimiento en la memoria de los adultos mayores ${ }^{29}$.

De hecho, al parecer la caminata resulta ser eficaz para mejorar el EC y la aptitud aeróbica, puesto que algunos estudios sugieren que las personas que comúnmente participan en cualquier tipo de actividad física regular muestran un mejor nivel cognitivo en relación a los que no realizan ningún tipo de actividad física ${ }^{30}$ e inclusive reflejan un mejor desempeño aerobico ${ }^{31}$.

Por lo tanto, estos hallazgos con intervenciones en entrenamiento aeróbico mediante caminatas, generalmente tienen un impacto más profundo en la salud cardiovascular en comparación con el entrenamiento de resistencia ${ }^{26}$, inclusive dicho entrenamiento es sugerido para mejorar los procesos del control y la atención selectiva en sujetos diabéticos ${ }^{32}$ y no diabéticos ${ }^{24}$.

En general, el programa de caminata en línea recta, propuesto en este estudio se basó en intensidades de baja a moderada, lo que depende principalmente del proceso de generación de energía aeróbica en referencia al uso de oxígeno para satisfacer adecuadamente las demandas de energía durante el ejercicio ${ }^{33}$. Además, todas las actividades aeróbicas, como caminar, correr, nadar, hacer gimnasia acuática, bicicleta, remar, entre otras, mejoran la salud cardiovascular, la rigidez arterial, el estrés oxidativo, la inflamación sistémica y el mejoramiento de la función endotelial ${ }^{34}$.

En consecuencia, los beneficios de la actividad física en general, pueden ayudar a las personas con diabetes a lograr una variedad de objetivos, por ejemplo, el aumento de la aptitud aérobica y función cognitiva como se observó en esta investigación. Otros estudios también han evidenciado el aumento de vigor, control glucémico mejorado, disminución de la resistencia a la insulina, mejoramiento del perfil lipídico, reducción de la presión arterial y mantenimiento de la pérdida de peso ${ }^{35,36}$.

Por lo tanto, los sujetos con diabetes mellitus pueden llegar a múltiples complicaciones cuando no se tratan a tiempo, especialmente si se encuentran físicamente inactivos durante el proceso de envejecimiento, lo que puede provocar disminución de las funciones físicas y mentales, así como provocar algunos efectos de las enfermedades crónicas para mantener a las personas mayores móviles e independientes ${ }^{37}$, puesto que el ejercicio físico por medio de caminatas controladas contribuye no solo a las adaptaciones crónicas, sino también al parecer a la plasticidad neuronal (cerebral estructural) durante la vejez.

El estudio presenta varias potencialidades, puesto que cumplió con el control de las fuentes de invalidación interna de los estudios experimentales puros. Por ejemplo, se mantuvo una tasa de retención del GE en $85 \%$, los sujetos fueron distribuidos por grupos de edad para evitar sesgos por el amplio rango de edades utilizados (maduración) y, además, los dos grupos fueron aleatorizados, con lo cual, todos los sujetos tuvieron la misma probabilidad de ser seleccionados. Además, es uno de los pocos estudios que presenta estas características metodológicas, lo cual, se advierte la generalización a otros contextos en sujetos con similares características al del presente estudio. También se reconoce, que no fue posible considerar en el experimento a varones, pues esta información hubiera permitido extender la generalización a ambos géneros, además, la evaluación de la aptitud aeróbica se efectuó por medio de una prueba indirecta de campo, por lo que los resultados deben ser analizados con precaución.

Este estudio demostró que un programa de caminatas en superficie plana, desarrollado tres veces por semana durante 48 sesiones, permitió contribuir al mejoramiento del EC y la aptitud aeróbica en mujeres con diabetes mellitus tipo 2. Estos resultados sugieren que los programas de actividad física en los sistemas de salud deben implantar la práctica cotidiana de la caminata como un medio de prevención de enfermedades metabólicas y de salud cognitiva durante el envejecimiento. Esta actividad puede servir como medicamento de bajo coste y es una alternativa para el deterioro cognitivo y cardiovascular.

\section{Referencias}

1. Fielding RA. The role of progressive resistance training and nutrition in the preservation of lean body mass in the elderly. J Am Coll Nutr 1995; 14: 587-94.

2. National Center for Chronic Disease Prevention and Health Promotion. Unrealized Prevention Opportunities: Reducing the Health and Economic Burden of Chronic Disease. Atlanta, GA: Centers for Disease Control and Prevention; 1997.

3. Sapunar J. Epidemiología de la diabetes mellitus en Chile. Diabetes 2016; 27 (2): 134-282. 
4. Guimarães A, Rocha S, Barbosa AR. Exercise and cognitive performance in older adults: a systematic review. Medicina (Ribeirão Preto) 2014; 47 (4): 377-86.

5. Chaddock L, Erickson KI, Prakash RS, Kim JS, Voss MW, Vanpatter M, et al. A neuroimaging investigation of the association between aerobic fitness, hippocampal volume, and memory performance in preadolescent children. Brain Res 2010; 1358: 172-83.

6. Miller DI, Taler V, Davidson PSR, Messier C. Measuring the impact of exercise on cognitive aging: methodological issues. Neurobiol Aging 2012; 33: 29-42.

7. Wang HX, MacDonald SWS, Dekhtyar S, Fratiglioni L. Association of lifelong exposure to cognitive reserve-enhancing factors with dementia risk: A community-based cohort study. PLoS Medicine 2017; 14 (3): e1002251.

8. Ismail I, Keating SE, Baker MK, Johnson NA. A systematic review and meta-analysis of the effect of aerobic vs. resistance exercise training on visceral fat. Obes Rev 2012; 13: 68-91.

9. Göçer E, Ardıç F, Akkaya N, Herek D. La eficacia de la caminata de intensidad moderada proporcionada por ECE PEDO sobre la grasa abdominal en mujeres con sobrepeso y obesidad: un estudio de ejercicio aleatorizado. Rev Turca Med Fis Rehabilit 2017; 63 (4): 340-7.

10. deJong A, Franklin BA. Prescribing exercise for the elderly: Current research and recommendations. Curr Sports Med Rep 2004; 3: 337-43.

11. Prohaska TR, Eisenstein AR, Satariano WA, Hunter R, Bayles CM, Kurtovich E, et al. Walking and the preservation of cognitive function in older populations. Gerontologist. 2009; 49 Suppl 1: S86-93.

12. Bielak AA, Cherbuin N, Bunce D, Anstey KJ. Preserved differentiation between physical activity and cognitive performance across young, middle, and older adulthood over 8 years. J Gerontol B Psychol Sci Soc Sci. 2014; 69 (4): 523-32.

13. Brach JS, VanSwearingen JM. Interventions to Improve Walking in Older Adults. Curr Transl Geriatr and Exp Gerontol Rep 2013; 2: 230-8.

14. Park JH, Miyashita M, Takahashi M, Kawanishi N, Hayashida $\mathrm{H}$, et al. Low-volume walking program improves cardiovascular-related health in older adults. J Sports Sci Med. 2014; 13 (3): 624-31.

15. Chou WT, Tomata Y, Watanabe T, Sugawara Y, Kakizaki M, Tsuji I. Relationships between changes in time spent walking since middle age and incident functional disability. Prev Med 2014; 59: 68-72.

16. Thomas A, Dennis A, Bandettini P, Johansen-Berg $H$. The effects of aerobic activity on brain structure. Front. Psychol 2012; 3: 86. https://doi.org/10.3389/ fpsyg.2012.00086.
17. Quiroga LP, Albala BC, Klaasen PG. Validación de un test de tamizaje para el diagnóstico de demencia asociada a edad, en Chile. Rev Med Chile 2004; 132: 467-78.

18. Folstein MF, Folstein SE, McHugh PR. "Mini-mental state". A practical method for grading the cognitive state of patients for the clinician. J Psychiatr Res. 1975; 12 (3): 189-98.

19. American Diabetes Association. (ADA). Diagnosis and Classification of Diabetes Mellitus. Diabetes Care 2014; 37 (Supplement 1): S81-90.

20. Mossberg KA, Fortini E. Responsiveness and validity of the six-minute walk test in individuals with traumatic brain injury. Physical Therapy 2012; 92 (5): 726-33.

21. Nolen-Doerr E, Crick K, Saha C, Groot M, Pillay Y, Shubrook J, et al. Six-Minute Walk Test as a Predictive Measure of Exercise Capacity in Adults with Type 2 Diabetes. Cardiopulm Phys Ther J 2018; 29 (3): 124-9.

22. Colegio Americano de Medicina del Deporte CAMD. Manual para la valoración y prescripción del ejercicio. Paidotribo. 3ra edición, 1986; Barcelona, España.

23. Cohen J. Statistical power analysis for the behavioral sciences (2nd ed.). Hillsdale, NJ, 1988; Lawrence Erlbaum.

24. Kramer AF, Hahn S, Cohen NJ, Banich MT, McAuley E, Harrison CR, et al. Ageing, fitness and neurocognitive function. Nature 1999; 400 (6743): 418-9.

25. Dumurgier J, Elbaz A, Ducimetière P, Tavernier B, Alpérovitch A, Tzourio C. Slow walking speed and cardiovascular death in well functioning older adults: prospective cohort study. BMJ 2009; 10: 339: b4460.

26. Rikli R, Edwards DJ. Effects of a three-year exercise program on motor function and cognitive processing speed in olderwomen. Res Q Exerc Sport 1991; 62 (1): 61-7.

27. Najafipour F, Mobasseri M, Yavari A, Nadrian H, Aliasgarzadeh A, Mashinchi Abbasi N, et al. Effect of regular exercise training on changes in $\mathrm{HbAlc}$, BMI and $\mathrm{VO}_{2}$ max among patients with type 2 diabetes mellitus: an 8-year trial. BMJ Open Diabetes Res Care. 2017; 5 (1): e000414.

28. Binu T, Uma Y, Benjamin T, Peiying L, Benjamin L, Rong Z, et al. Life-long aerobic exercise preserved baseline cerebral blood flow but reduced vascular reactivity to $\mathrm{CO}_{2}$. J Magn Reson Imaging 2013; 38: 1177-83.

29. Erickson KI, Prakash RS, Voss MW, Chaddock L, Hu $\mathrm{L}$, Morris KS, et al. Aerobic fitness is associated with hippocampal volume in elderly humans. Hippocampus 2009; 19 (10): 1030-9.

30. Aichberger MC, Busch MA, Reischies FM, Strohle A, Heinz A, Rapp MA. Effect of physical inactivity on cognitive performance after 2.5 years of follow-up: lon- 
Ejercicio físico sobre aptitud aeróbica en mujeres mayores - E. Molina-Sotomayor et al

gitudinal results from the survey of health, ageing, and retirement (SHARE). GeroPsych 2010; 23 (1): 7-15.

31. Busse AL, Gil G, Santarém JM, Jacob Filho W. Actividad física y cognición en los ancianos: una revisión. Demencia y neuropsicología 2009; 3 (3): 204-8.

32. Nielsen PJ, Hafdahl AR, Conn VS, Lemaster JW, Brown SA. Meta-analysis of the effect of exercise interventions on fitness outcomes among adults with type 1 and type 2 diabetes. Diabetes Res Clin Pract 2006; 74: 111-20.

33. Cammisuli AM, Innocenti A, Franzoni F, Pruneti C. Aerobic Exercise Effects upon Cognition in Mild Cognitive Impairment: a Systematic Review of Randomized Controlled Trials. Archives Italiennes de Biologie 2017; 155: 54-62. Doi 10.12871/000398292017126.
34. Forbes D, Thiessen EJ, Blake CM, Forbes SC, Forbes S. Exercise programs for people with dementia. Cochrane Database Systematic Reviews 2013; 12: CD006489.

35. Chudyk A, Petrella RJ. Effects of exercise on cardiovascular risk factors in type 2 diabetes: A meta-analysis. Diabetes Care 2011; 34: 1228-37.

36. Colberg SR, Sigal RJ, Yardley JE, Riddell MC, Dunstan DW, Dempsey PC. Physical activity/exercise and diabetes: A position statement of the American Diabetes Association. Diabetes Care 2016; 39: 2065-79.

37. McPhee JS, French DP, Jackson D, Nazroo J, Pendleton N, Degens H. Physical activity in older age: perspectives for healthy ageing and frailty. Biogerontology 2016; 17 (3): 567-80. 